\title{
Primary Cutaneous Adenoid Cystic Carcinoma of Scalp
}

\author{
${ }^{1}$ Arvind Krishnamurthy, ${ }^{2}$ Anitha Vaidhyanathan \\ ${ }^{1}$ Associate Professor, Department of Surgical Oncology, Cancer Institute (WIA), Adayar, Chennai, Tamil Nadu, India \\ ${ }^{2}$ Observer, Department of Surgical Oncology, Cancer Institute (WIA), Adayar, Chennai, Tamil Nadu, India
}

Correspondence: Arvind Krishnamurthy, Associate Professor, Department of Surgical Oncology, Cancer Institute (WIA) 36 Sardar Patel Road, Adayar, Chennai-20, Tamil Nadu, India, Phone: 9840448174, e-mail: drarvindkrishnamurthy@yahoo.co.in

\begin{abstract}
The scalp is a unique anatomical site that can be involved with a variety of malignant and premalignant conditions, although a majority of the lesions are benign. Adenoid cystic carcinoma is most commonly seen as a neoplasm of the salivary glands and seromucinous glands of the upper respiratory tract. Primary cutaneous adenoid cystic carcinoma (PCACC) is a rare appendageal tumor should only be considered if there is no history or evidence of extracutaneous adenoid cystic carcinoma. It usually has an indolent course but has a high tendency to recur locally. Lymph node and distant metastasis are exceedingly rare with only a handful of cases being reported. We report a case of a large scalp PCACC in a 61 years old gentleman with lymph node involvement at presentation.

Keywords: Primary cutaneous adenoid cystic carcinoma (PCACC), scalp tumors.
\end{abstract}

\section{INTRODUCTION}

Adenoid cystic carcinoma (ACC) in its commonest presentation is as a neoplasm of the salivary glands. It is also known to occur in the lacrimal glands, mucosal glands of the upper respiratory tract, external auditory canal, breast, Bartholin's glands of the vulva, uterine cervix, prostate gland, esophagus, and other sites. ${ }^{1}$ The skin can also be involved with ACC through direct or perineural invasion or as a site of distant metastases. ${ }^{2}$ However, in contradistinction, primary cutaneous adenoid cystic carcinoma (PCACC) is a rare entity characterized by an indolent but progressive course, a tendency to recur locally, and rare nodal or metastatic spread. ${ }^{3}$ Despite its limited potential for early metastases, the tumor exhibits aggressive infiltrative growth in the reticular dermis and subcutis with frequent perineural invasion. This explains perhaps the reasons behind markedly high-recurrence rates (50\%). ${ }^{1,4}$

We present a unique case of an aggressive PCACC of the scalp metastatic to cervical lymph nodes.

\section{CASE REPORT}

A 61 years old gentleman presented with a large nodular swelling of his scalp gradually increasing since 12 years. A recent increase in size with the onset of pain prompted him to seek medical attention at an outside clinic where in an

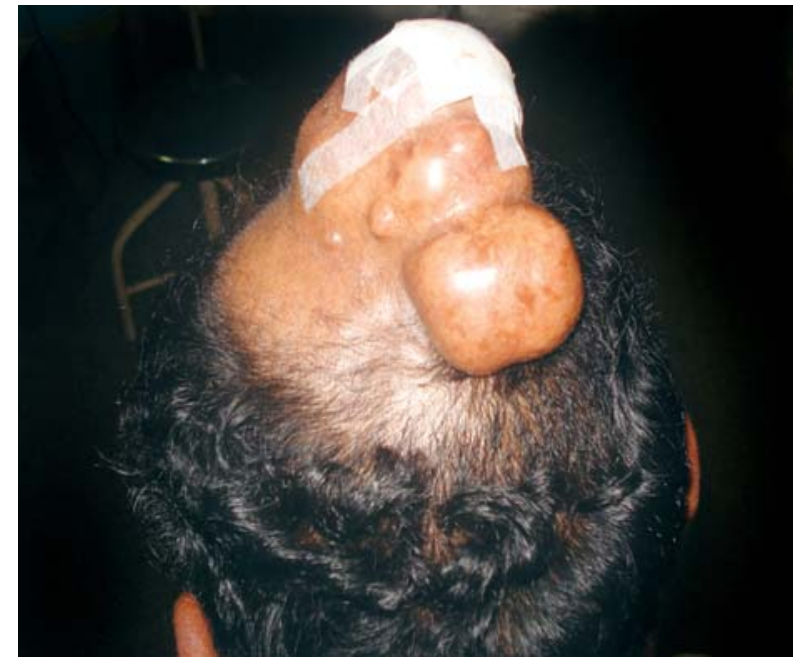

Fig. 1: Tumor at time of presentation

incisional biopsy was done (Fig. 1). The slides reviewed at our center showed features suggestive of Adenoid Cystic Carcinoma. He had comorbid illnesses in the form of hypertension and ischemic heart disease which was controlled with cardiac medication.

Local examination revealed a $10 \times 5 \mathrm{~cm}$ irregular nodular swelling in the midline and right posterior parietal region of the skin of the scalp with multiple confluent nodules and a surface ulceration $2 \times 2 \mathrm{~cm}$ at the site of the incisional biopsy. A $1 \times 0.5 \mathrm{~cm}$ firm node was palpable in the right 


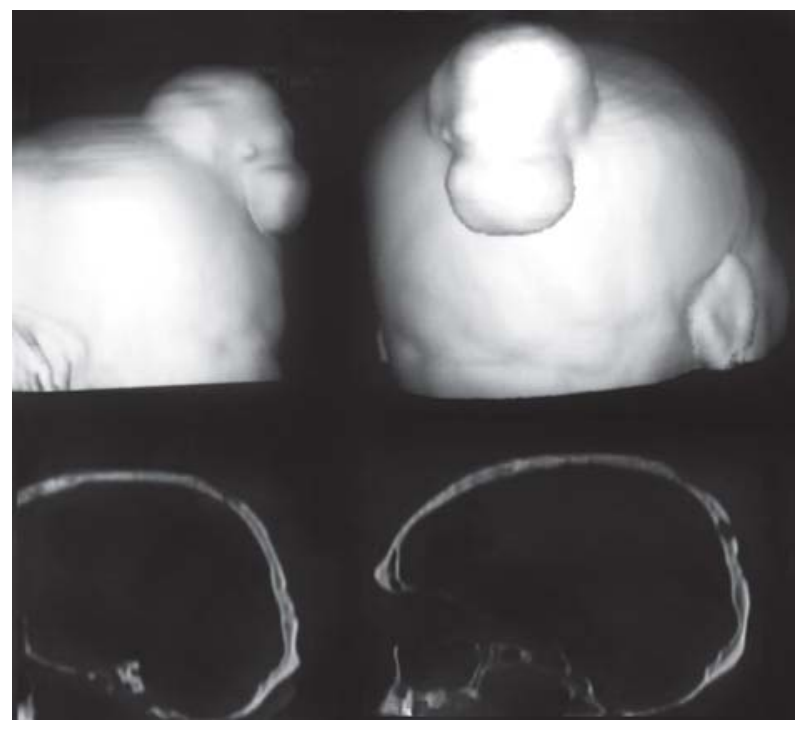

Fig. 2: Cross-section CT scan image with reconstruction

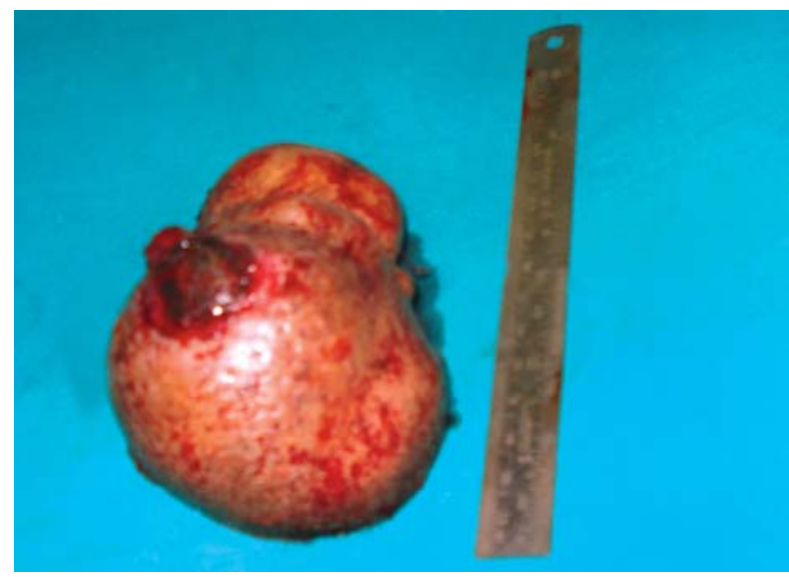

Fig. 3: Specimen of wide excision of scalp tumor

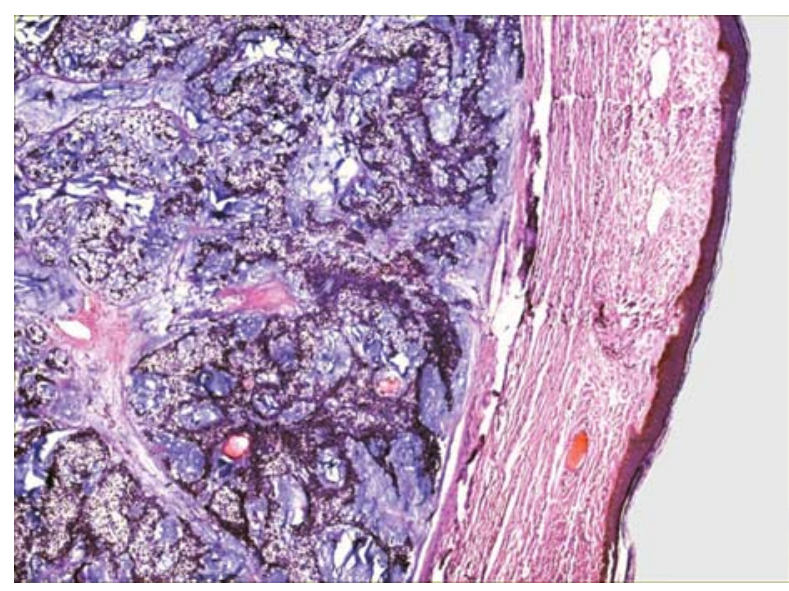

Fig. 4: Skin with the dermis showing tumor arranged in a cribriform pattern H\&E $4 \mathrm{X}$

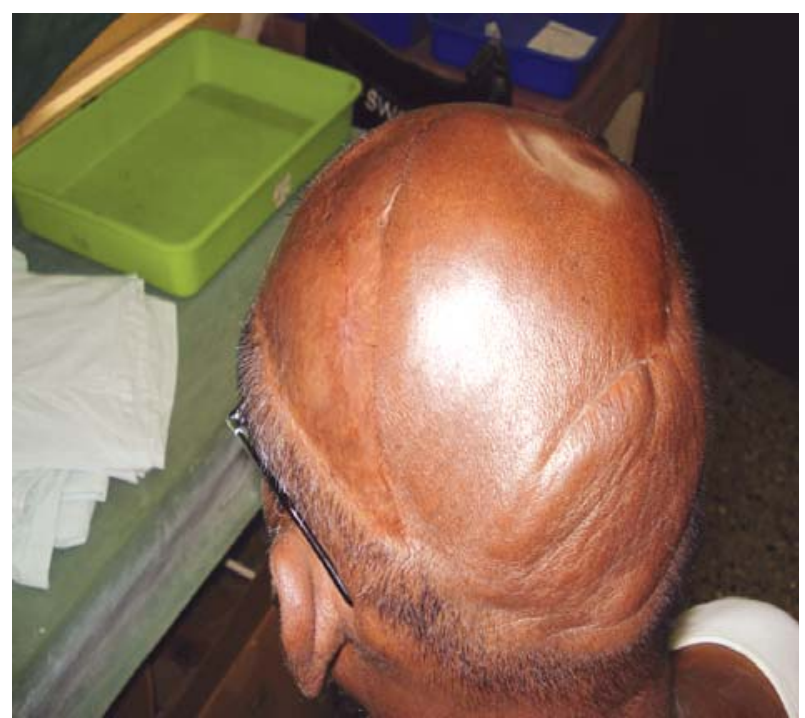

Fig. 5: The patient 2 years following treatment completion

posterior auricular region. A comprehensive examination of the aerodigestive tract, including the salivary glands was unremarkable.

A CT scan of the head and neck revealed the swelling involving the full thickness of the scalp with involvement of the outer table of the cranium (Fig. 2). A chest skiagram was normal. He underwent a wide local excision of the scalp tumor with removal of the overlying outer table of skull along with right posterolateral neck dissection. The scalp defect was reconstructed using a local scalp rotation flap.

Grossly, the tumor consisted of several elevated, confluent nodules (Fig. 3). The final histopathology was reported as a $10 \times 5.5 \times 5 \mathrm{~cm}$ poorly circumscribed tumor which on microscopy revealed solid epithelial sheets in the reticular dermis and areas of cribriform islands consisting of basaloid cells with uniform round spaces containing myxoid material. Tumor cells had a uniform character and mitosis was rare. The positive immunohistochemical markers of the current case were muscle actin, vimentin, calponin and S-100. The final impression was that of a cribriform variant of Adenoid Cystic Carcinoma with free margins, 4 out of 24 nodes harbored metastatic deposits. No perineural invasion was made out (Fig. 4).

He received External Beam Radiation to his scalp and neck and is currently disease free for more than two years (Fig. 5).

\section{DISCUSSION}

The scalp is a unique anatomical site that can be involved with a variety of malignant and premalignant conditions. 
Although majority of the lesions diagnosed, remain benign, the evaluation of head masses involves an orderly approach based on a careful history and accurate physical examination. Certain lesional characteristics, such as pain, a hard consistency, immobility or a large size might need further evaluation using appropriate laboratory and radiological investigations. $^{5}$

PCACC originating in the scalp was first reported by Boggio in $1975 .{ }^{6}$ Despite its clinical recognition for over 35 years, the etiology, origins and histogenesis of PCACC is poorly understood and there appears to be no universally agreed predisposing factor.

PCACC most commonly arises in the sixth and seventh decades of life with no specific sex prediliction. The average duration of the tumor prior to diagnosis is 9.8 years with a tumor size ranging from 0.5 to $8.0 \mathrm{~cm}$ with an average size of $3.2 \mathrm{~cm} .{ }^{7}$ PCACC appears clinically as a firm, poorly circumscribed, slow growing nodule. The tumor most often occupies an intradermal and subcutaneous location, with $40 \%$ of PCACCs arising on the scalp. Other reported locations include the chest, abdomen, back, axilla, eyelid, and perineum. ${ }^{1}$ Patients may be asymptomatic or may present with local hair loss, pain, tenderness, or rarely, pruritus. ${ }^{3,6}$ It is however, reasonable to rule out a direct cutaneous spread or a cutaneous metastasis of an adenoid cystic carcinoma of the salivary glands before a diagnosis of PCACC is made. Lymph node and pulmonary metastases have been reported in a total of 5 and 8 cases respectively. ${ }^{7}$

The unique features of our case were the large tumor size and the presence of multiple lymph nodal metastases. However, on all other counts, our case was typical for PCACC given the location, age of the patient, presenting clinical features, and most importantly microscopy and immunohistochemical findings.

The primary modality of management is surgery, which involves wide local excision with tumor-free margins is the treatment of choice. Discontinuous perineural involvement may produce false-negative margins leading to inadequate resection. ${ }^{1}$ Even excision with $2 \mathrm{~cm}$ margins has been reported to be positive for neoplastic cells in the excisional margin. There are also reports of PCACC treated with Mohs surgery. ${ }^{8}$

Resection of regional lymph nodes is not recommended in cases without clinical evidence of lymph node metastases as in all published reports. ${ }^{1,6,7,9,10}$

External beam radiation therapy has been used in the adjuvant setting in some cases. Some authors advocate addition of chemotherapy as well. Combination cisplatin and fluorouracil chemotherapy has been successfully used to treat PCACC with pulmonary metastases. ${ }^{11}$

PCACC characteristically follows an indolent course but has a high tendency to recur locally after surgery. Reported intervals for recurrences have ranged from 1 month to 35 years with extended tumor-free interval indicating the need for long-term follow-up. Naylor et al found $44 \%$ of reported PCACC cases to recur with an average of 58 months of follow-up.

\section{CONCLUSION}

PCACC are generally considered low grade carcinomas, nodal and distant metastasis is very rare. The delay in seeking prompt medical attention on the part of the patient possibly resulted in the tumor progression in our case.

\section{REFERENCES}

1. Salzman M, Eades E. Primary cutaneous adenoid cystic carcinoma: A case report and review of the literature. Plast Reconstr Surg 1991;88(1):140-44.

2. King DT, Cihak RW, Luther PK, Gurevitch AW, Hirose FM. Malignant neoplasms of the paranasal sinuses involving the skin. Arch Dermatol Nov 1978;114(11):1681-83.

3. Naylor E, Sarkar P, Perlis CS, Giri D, Gnepp DR, RobinsonBostom L. Primary cutaneous adenoid cystic carcinoma. J Am Acad Dermatol 2008;58:636-41.

4. Pappo O, Gez E, Craciun I, Zajicek G, Okon E. Growth rate analysis of lung metastases appearing 18 years after resection of cutaneous adenoid cystic carcinoma. Arch Pathol Lab Med 1992;116:76-79.

5. Baldwin HE, Clifford MB, Yelva LL. J Am Acad Dermatol 1991;25:819-30.

6. Boggio R. Adenoid cystic carcinoma of the scalp. Arch Dermatol 1975;111:793-94.

7. Kato N, Yasukawa K, Onozuka T. Primary cutaneous adenoid cystic carcinoma with lymph node metastasis. Am J Dermatopathol 1998;20:571-77.

8. Krunic AL, Kim S, Medenica M, Laumann AE, Soltani K, Shaw JC. Recurrent adenoid cystic carcinoma of the scalp treated with mohs micrographic surgery. Dermatol Surg Jun 2003;29(6): 647-49.

9. Cooper PH, Adelson GL, Holthaus WH. Primary cutaneous adenoid cystic carcinoma Arch Dermatol Jun 1984;120(6): 774-77.

10. Weekly M, Lydiatt DD, Lydiatt WM, Baker SC, Johansson SL. Primary cutaneous adenoid cystic carcinoma metastatic to cervical lymph nodes. Head Neck 2000;22:84-86.

11. Singh A, Ramesh V. Primary cutaneous adenoid cystic carcinoma with distant metastasis: A case report and brief literature review. Indian J Dermatol Venereol Leprol 2010; 76:176-79. 